\title{
Comparison of a Photon-Counting-Detector CT with an Energy- Integrating-Detector CT for Temporal Bone Imaging: A Cadaveric Study
}

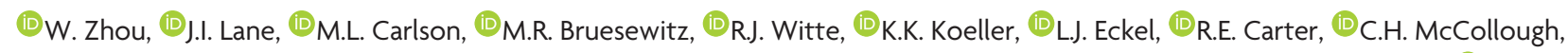
and ${ }^{1}$ S. Leng

\begin{abstract}
BACKGROUND AND PURPOSE: Evaluating abnormalities of the temporal bone requires high-spatial-resolution CT imaging. Our aim was to assess the performance of photon-counting-detector ultra-high-resolution acquisitions for temporal bone imaging and compare the results with those of energy-integrating-detector ultra-high-resolution acquisitions.
\end{abstract}

MATERIALS AND METHODS: Phantom studies were conducted to quantify spatial resolution of the ultra-high-resolution mode on a prototype photon-counting-detector CT scanner and an energy-integrating-detector CT scanner that uses a comb filter. Ten cadaveric temporal bones were scanned on both systems with the radiation dose matched to that of the clinical examinations. Images were reconstructed using a sharp kernel, 0.6- $\mathrm{mm}$ (minimum) thickness for energy-integrating-detector CT, and 0.6- and 0.25-mm (minimum) thicknesses for photon-counting-detector CT. Image noise was measured and compared using adjusted 1-way ANOVA. Images were reviewed blindly by 3 neuroradiologists to assess the incudomallear joint, stapes footplate, modiolus, and overall image quality. The ranking results for each specimen and protocol were compared using the Friedman test. The Krippendorff $\alpha$ was used for interreader agreement.

RESULTS: Photon-counting-detector CT showed an increase of in-plane resolution compared with energy-integrating-detector CT. At the same thickness $(0.6 \mathrm{~mm})$, images from photon-counting-detector CT had significantly lower $(P<.001)$ image noise compared with energy-integrating-detector CT. Readers preferred the photon-counting-detector CT images to the energy-integrating-detector images for all 3 temporal bone structures. A moderate interreader agreement was observed with the Krippendorff $\alpha=0.50$. For overall image quality, photon-counting-detector CT image sets were ranked significantly higher than images from energy-integrating-detector $\mathrm{CT}$ $(P<.001)$.

CONCLUSIONS: This study demonstrated substantially better delineation of fine anatomy for the temporal bones scanned with the ultra-high-resolution mode of photon-counting-detector CT compared with the ultra-high-resolution mode of a commercial energyintegrating-detector CT scanner.

ABBREVIATIONS: EID $=$ energy-integrating detector; $\mathrm{K}-\alpha=$ Krippendorff $\alpha ;$ MTF $=$ modulation transfer function; $\mathrm{PCD}=$ photon-counting detector; UHR $=$ ultra-high-resolution

M ultidetector CT is an essential clinical diagnostic tool for evaluating abnormalities of the temporal bone and lateral skull base. ${ }^{1-4}$ Temporal bone structures of clinical interest, such as

\footnotetext{
Received March 13, 2018; accepted after revision June 19.

From the Departments of Radiology (W.Z., J.I.L., M.R.B., R.J.W., K.K.K., L.J.E., C.H.M., S.L.) and Otolaryngology-Head and Neck Surgery (M.L.C.) and Division of Biomedical Statistics and Informatics (R.E.C.), Mayo Clinic, Rochester, Minnesota.

Research reported in this article was supported by the National Institutes of Health under award numbers R01 EB016966 and C06 RR018898 and in collaboration with Siemens.

The content is solely the responsibility of the authors and does not necessarily represent the official views of the National Institutes of Health.

Please address correspondence to Shuai Leng, PhD, Department of Radiology, Mayo Clinic, 200 First St SW, Rochester, MN 55905; e-mail: leng.shuai@mayo.edu

- Indicates open access to non-subscribers at www.ajnr.org

http://dx.doi.org/10.3174/ajnr.A5768
}

the ossicles, facial nerve, and labyrinth, are submillimeter and require high-spatial-resolution imaging. ${ }^{2,5}$ The detector size of a CT system is one of the major factors limiting the spatial resolution needed to resolve these fine structures. Commercially available multidetector CT scanners are built using energy-integrating detectors (EIDs), in which the detected signal is proportional to the total energy deposited by all photons without specific information about an individual photon or its energy. The effective detector pixel sizes range from 0.5 to $0.625 \mathrm{~mm}$ at the isocenter for the commercial EIDs. Several approaches have been investigated to further improve the spatial resolution of an EID system for temporal bone imaging. One approach is to place an attenuating comb (grid) filter on top of the detector to reduce the detector aperture size. ${ }^{6-8}$ However, the attenuation of the filter inevitably reduces geometric dose efficiency because the filter blocks the 
photons after they have passed through the patient., ${ }^{6,9}$ Other methods, such as using a flat panel detector, do not satisfy the clinical requirements for contrast-to-noise ratio, scan field of view, or temporal resolution. ${ }^{10,11}$

A whole-body photon-counting detector (PCD) CT scanner has been installed in our laboratory for research use (Somatom CounT; Siemens, Erlangen, Germany) ${ }^{12-14}$ and is not yet commercially available. Different from the conventional EIDs that integrate deposited energies from all photons, PCDs use directconversion techniques and count individual photons while measuring energy information. Studies have demonstrated many benefits of PCDs over the conventional EIDS, such as less impact of electronic noise, a higher contrast-to-noise ratio, improved dose efficiency, and simultaneous multi-energy imaging. ${ }^{15-22}$ PCDs can eliminate the septa between adjacent detector pixels required by EIDs to avoid cross-talk and maintain spatial resolution. This feature leads to a PCD detector size of $0.25 \mathrm{~mm}$ at the isocenter (compared with $0.5-0.6 \mathrm{~mm}$ for EIDs) without compromising dose efficiency. To date, preliminary phantoms and cadaveric studies have reported $150-\mu \mathrm{m}$ limiting spatial resolution for this scan mode and have demonstrated the potential benefits of superior image quality from ultra-high-resolution (UHR) PCD-CT acquisitions. ${ }^{23,24}$ However, none of the studies has evaluated specific clinical tasks and investigated how radiologists' reading performance could benefit from the higher resolution capability of PCD-CT. Therefore, the purpose of this study was to assess the performance of PCD-CT UHR acquisitions for temporal bone imaging and compare the results with those of EID-CT UHR acquisitions.

\section{MATERIALS AND METHODS}

\section{Phantom Experiments to Evaluate Spatial Resolution}

A 50- $\mu \mathrm{m}$ diameter tungsten wire inserted into a solid water phantom was scanned along the $\mathrm{z}$-axis on the whole-body PCD-CT scanner using the UHR scan mode. The PCD-CT system was built on the platform of a second-generation dual-source CT scanner (Somatom Definition Flash; Siemens). Detailed descriptions of this system have been reported elsewhere. ${ }^{12-14}$ The UHR acquisition on the PCD-CT system has an effective pixel size of $0.25 \times$ $0.25 \mathrm{~mm}$ at the isocenter. ${ }^{23} \mathrm{PCD}-\mathrm{CT}$ scans were obtained with the following parameters: spiral mode, $120-\mathrm{kV}$ tube potential, $25-$ and $75-\mathrm{keV}$ energy thresholds, $32 \times 0.25 \mathrm{~mm}$ collimation, 0.8 pitch, and 1.0-second rotation time. For comparison, the wire phantom was also scanned on a second-generation dual-source CT scanner, the same platform on which the PCD-CT was built. Both the PCD-CT and EID-CT systems involved in this study use an identical UHR focal spot size of $0.7 \mathrm{~mm} .{ }^{25}$ EID-CT scans were obtained using the standard clinical protocol: UHR mode with a comb filter along the fan direction, spiral mode, $120-\mathrm{kV}$ tube potential, $16 \times 0.6 \mathrm{~mm}$ collimation, 0.8 pitch, and 1.0 -second rotation time. All images were reconstructed with a standard weighted filtered back-projection algorithm, 0.6-mm slice thickness, and a sharp kernel (U70). Modulation transfer function (MTF) is commonly used to provide a comprehensive evaluation of spatial resolution for imaging systems by assessing the system response with respect to the input signal at each frequency. In this study, the MTF was calculated from the point spread function of the wire in the axial images to assess the in-plane spatial resolution. Spatial frequencies of $50 \%, 10 \%$, and $2 \%$ MTF values were recorded.

\section{Temporal Bone Specimens}

Ten formalin-fixed cadaveric temporal bone specimens were harvested by the department of anatomy using the block technique described by Schuknecht. ${ }^{26}$ All specimens had no known history of a prior operation or trauma and were otherwise anatomically intact. Each specimen was placed within a 20 -cm-diameter solidwater ring and placed in the supine position to replicate clinical temporal bone CT examinations.

\section{Imaging Protocol for Specimens}

Each specimen was scanned using the UHR mode with the same imaging protocols for phantom experiments on PCD-CT and EID-CT systems, respectively. Automatic exposure control was off and effective milliampere-second was set to match the radiation dose of clinical examinations (volume CT dose index $=61$ $\mathrm{mGy}$ ). All images were reconstructed using the weighted filtered back-projection method with a sharp kernel (U70). For PCD-CT acquisitions, image thickness and increment were set to $0.6 / 0.3$ and $0.25 / 0.25 \mathrm{~mm}$ (thinnest available), respectively. Image thickness and increment for the EID system were set to $0.6 / 0.3 \mathrm{~mm}$ (thinnest available). For simplification, the $3 \mathrm{CT}$-acquisition protocols are denoted as "detector type-image thickness" (in millimeters) (ie, PCD-0.6, PCD-0.25, and EID-0.6).

\section{Noise Measurements}

Image noise was measured in the cadaveric images as the standard deviation (SD) of CT numbers in a circular ROI drawn in a uniform soft-tissue area for each dataset. The size and the location of the ROIs were matched among the 3 image sets (PCD-0.6, PCD0.25 , and EID-0.6). The mean and SD of image noise for each image set were calculated.

\section{Reader Assessment of Image Quality}

The reading protocol was established on a clinical viewing station that was appropriately calibrated for routine diagnosis following the "ACR-AAPM-SIIM Technical Standard for Electronic Practice of Medical Imaging." "27 The 3 image sets (PCD-0.6, PCD0.25, and EID-0.6) for each temporal bone specimen were displayed side by side in a random order with scanning and reconstruction information blinded to the readers. Three fellowship-trained neuroradiologists (R.J.W., K.K.K., L.J.E.), each with $>10$ years of experience, independently assessed the overall image quality and the delineation of 3 anatomic structures (modiolus, stapes footplate, incudomallear joint). For each specimen, images from 3 protocols were ranked from 1 to 3, with 1 being the most preferred and 3 being the least preferred. Equal rank was allowed. Thirty sets of images ( 10 specimens $\times 3$ image sets/specimen) were reviewed by each of the 3 readers.

\section{Statistical Analysis}

All statistical analyses were performed using free statistical software (R Project, Version 3.4.0; http://www.r-project.org/). The differences of image noise among the 3 protocols were evaluated 
using 1-way ANOVA with subsequent Tukey honest significant difference analysis. The average ranking from the 3 readers for each specimen and protocol was compared using the Friedman test to evaluate the differences in overall image quality and diagnostic confidence for the 3 structures. Pair-wise comparisons were performed with Conover post hoc testing with a Bonferroni correction. $P<.05$ was considered statistically significant. The Krippendorff $\alpha(\mathrm{K}-\alpha)$ was used to test the interreader agreement with the following scales: $0-.20=$ poor agreement, $0.21-0.40=$ fair agreement, $0.41-0.60=$ moderate agreement, $0.61-0.80=$ substantial agreement, and $0.81-1.00=$ almost perfect agreement. ${ }^{28}$

\section{RESULTS}

PCD-CT showed a slightly better MTF performance than EIDCT (Fig 1). The spatial frequencies at 50\%, 10\%, and 2\% MTF

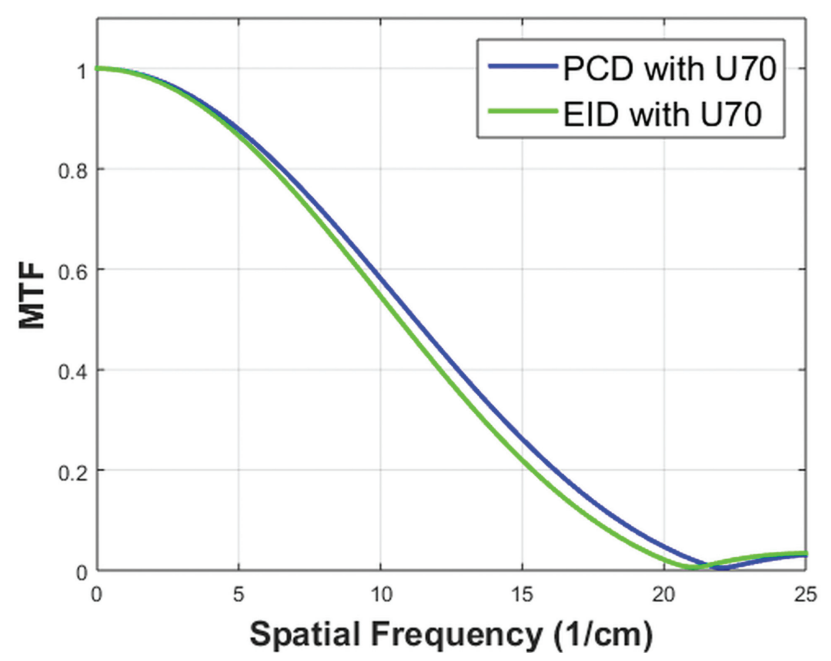

FIG 1. Comparison of MTF curves for UHR modes reconstructed with a sharp kernel (U70) on the PCD-CT and EID-CT systems.

\begin{tabular}{|c|c|c|c|}
\hline MTF & $50 \%$ & $10 \%$ & $2 \%$ \\
\hline PCD-CT with U70 kernel & $11.2 / \mathrm{cm}$ & $18.4 / \mathrm{cm}$ & $21.1 / \mathrm{cm}$ \\
\hline EID-CT with U70 kernel & $10.6 / \mathrm{cm}$ & $17.5 / \mathrm{cm}$ & $20.1 / \mathrm{cm}$ \\
\hline
\end{tabular}

(Table 1) were $11.2,18.4$, and $21.1 / \mathrm{cm}$ for the PCD-CT and 10.6 , 17.5 , and $20.1 / \mathrm{cm}$ for EID-CT.

Representative images of the modiolus (Fig 2), stapes footplate (Fig 3), and incudomallear joint (Fig 4), shown side-by-side for the 3 datasets (PCD-0.6, PCD-0.25, and EID-0.6), demonstrated the improved ability to resolve each of the evaluated structures. Decreased image thickness resulted in enhanced visualization of the 3 submillimeter structures evaluated.

Measurements for the same image thickness $(0.6 \mathrm{~mm}$, Fig 5) showed that images from the PCD scanner had significantly lower $(P<.001)$ image noise (mean, $55.9 \pm 5.2 \mathrm{HU})$ compared with images from the EID scanner (mean, $91.8 \pm 6.5 \mathrm{HU}$ ). The thinner 0.25-mm PCD images (mean, $89.8 \pm 8.3 \mathrm{HU}$ ) yielded noise like that of the 0.6-mm EID images $(P=.80)$.

The rank distributions from all 3 readers demonstrated that PCD-0.25 images were the most preferred, followed by the PCD0.6 images; the EID-0.6 images were the least preferred (Fig 6). The Friedman test showed statistically significant differences in rankings for the 3 protocols $(P=.02)$. Pair-wise comparison demonstrated that the readers preferred the PCD-CT images to the EID images for all 3 temporal bone structures (Table 2). Among the 3 sets of PCD images, readers preferred the PCD- 0.25 images over the PCD-0.6 images for visualizing the modiolus $(P=.002)$ and the incudomallear joint $(P<.001)$, but no significant preference was found when assessing the stapes footplates $(P=.12)$. For overall image quality, both PCD-CT image sets were ranked significantly higher than the EID images $(P<.001)$, and readers preferred thinner images $(0.25 \mathrm{~mm})$ over thicker images $(0.6 \mathrm{~mm})$ from PCD-CT $(P<.001)$.

Fair-to-moderate interobserver agreement was observed among the 3 readers for ranking image quality (Table 3 ). Readers reached moderate agreement for the modiolus $(\mathrm{K}-\alpha=0.54)$ and stapes footplate $(\mathrm{K}-\alpha=0.44)$ and fair agreement for the incudomallear joint $(\mathrm{K}-\alpha=0.36)$. For overall image quality, moderate agreement was observed with $\mathrm{K}-\alpha=0.50$.

\section{DISCUSSION}

In this in vitro study, we investigated temporal bone imaging using a new PCD-CT system with a $0.25 \times 0.25 \mathrm{~mm}$ detector size at its isocenter. Quantitative and qualitative image quality analyses
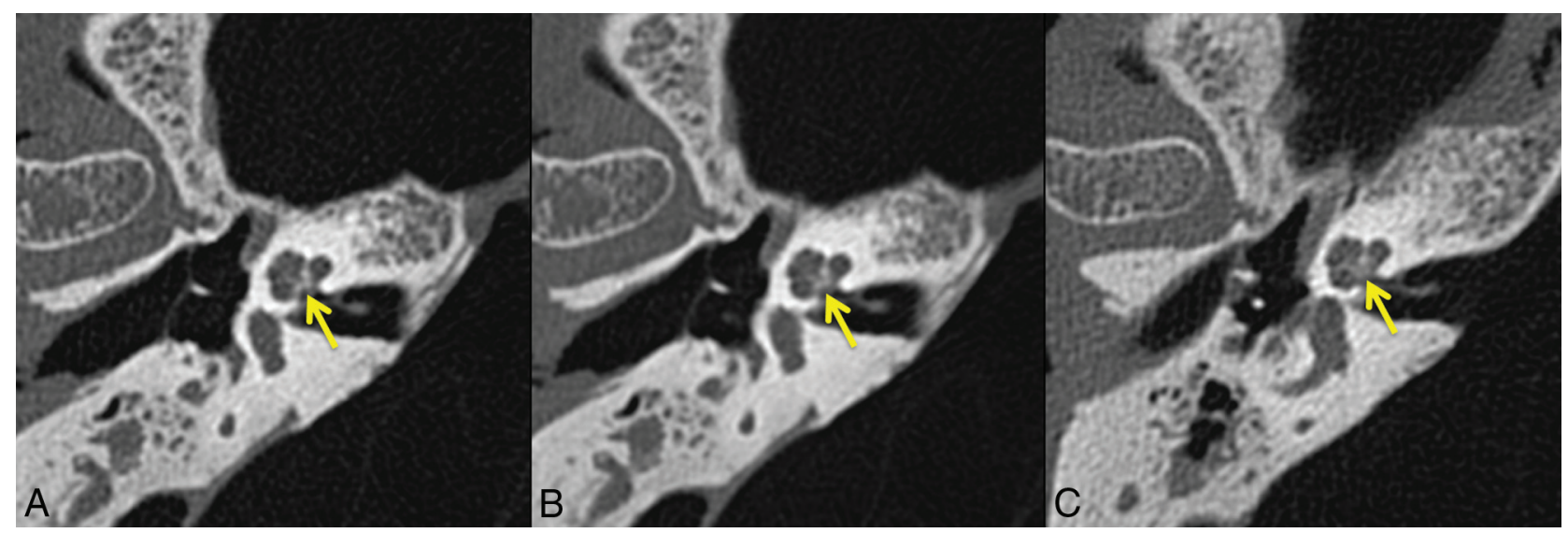

FIG 2. Representative axial images of the modiolus (arrow) from the same specimen scanned with UHR PCD-CT and reconstructed with 0.25 $(A)$ and 0.6-mm (B) image thicknesses, and UHR EID-CT, with a 0.6-mm image thickness (C). The pyramid-shaped modiolus is better depicted with the PCD-CT. 


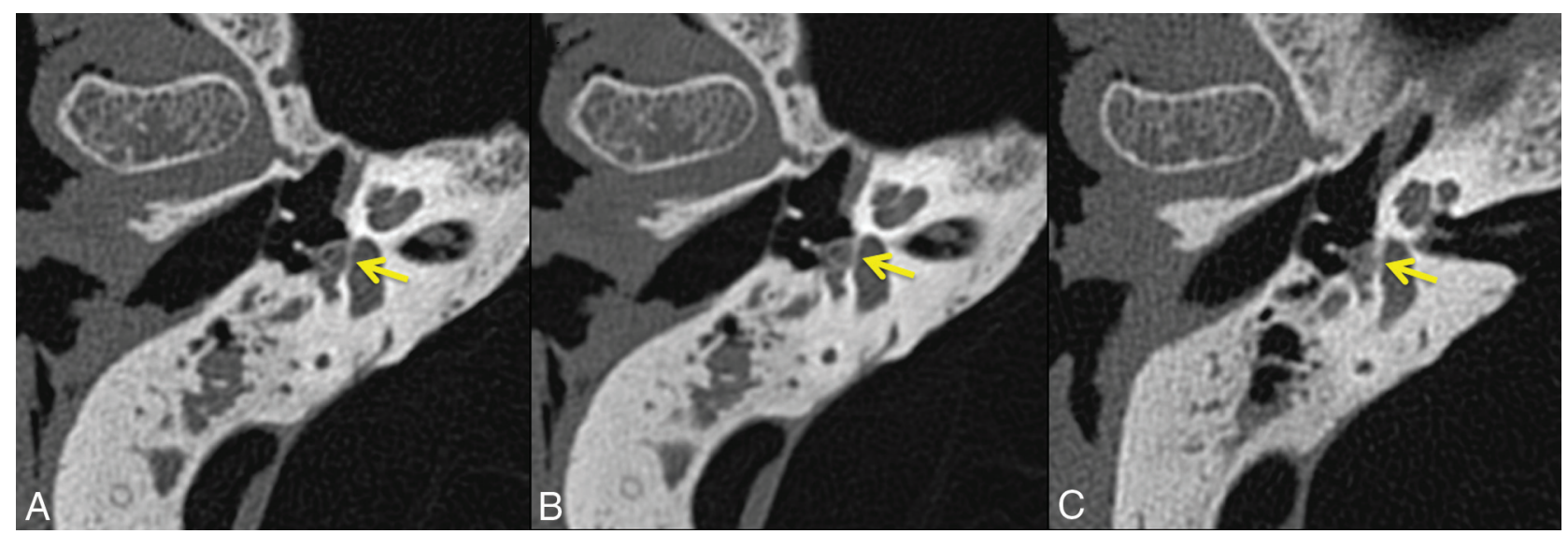

FIG 3. Representative axial images of the stapes footplate (arrow) from the same specimen scanned with UHR PCD-CT and reconstructed with $0.25-(A)$ and 0.6- $\mathrm{mm}(B)$ image thicknesses, and UHR EID-CT, with 0.6-mm image thickness (C). An improved illustration of the stapes footplate and the limbs of the stapes is observed for PCD-CT.

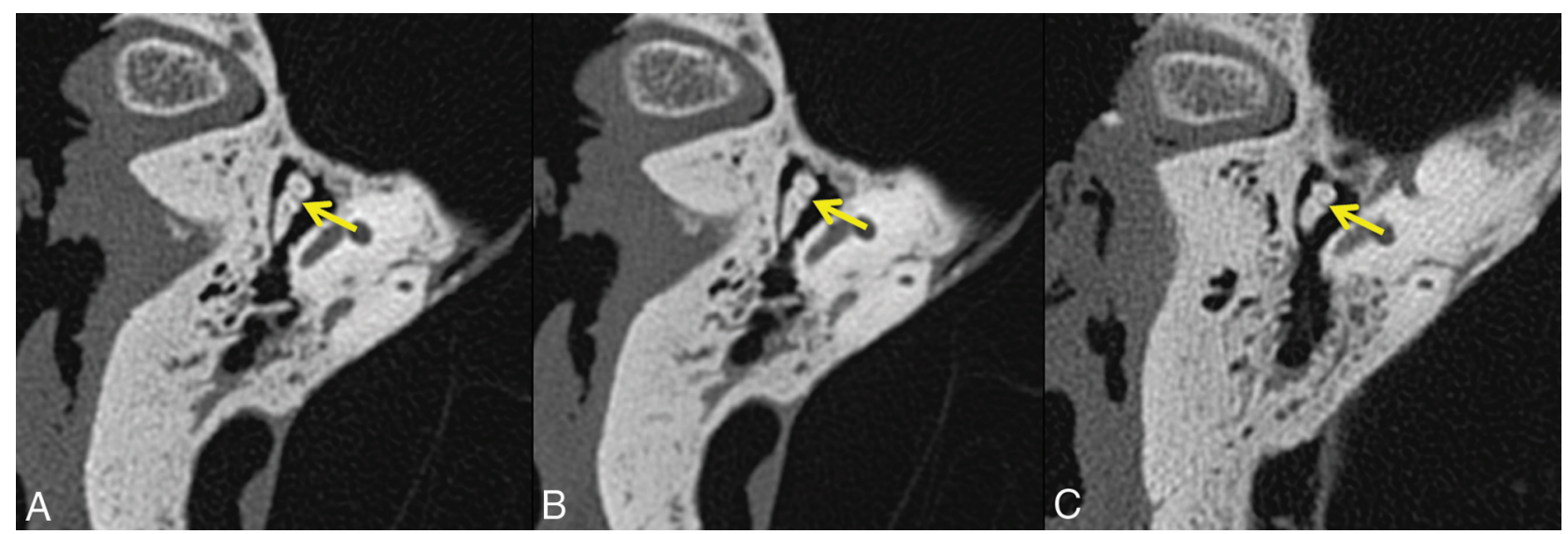

FIG 4. Representative axial images of the incudomallear joint (arrow) from the same specimen scanned with UHR PCD-CT and reconstructed with $0.25-(A)$ and 0.6-mm (B) image thicknesses, and UHR EID-CT, with 0.6-mm image thickness $(C)$. The incudomallear joint between the incus and malleus is better defined in PCD-CT images compared with EID-CT images.

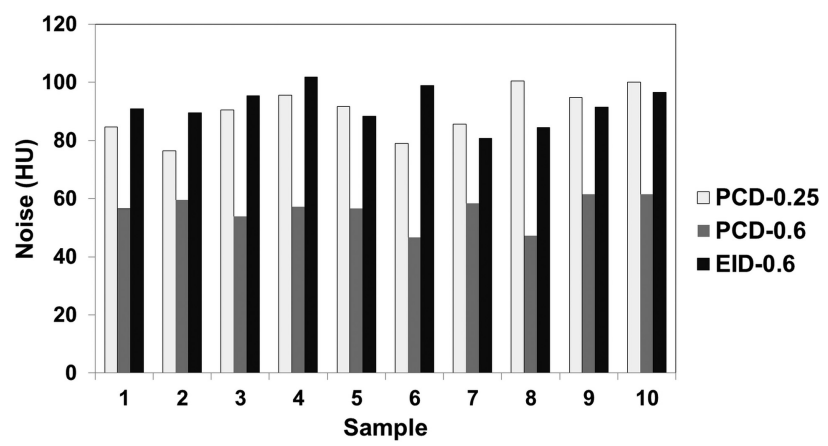

FIG 5. Image noise measured from 10 cadaveric specimens scanned with 3 UHR protocols. White indicates PCD with a $0.25-\mathrm{mm}$ image; gray, PCD with a 0.6-mm image; black, EID with a $0.6-\mathrm{mm}$ image.

showed superior image quality and better delineation of anatomic microstructures compared with the EID-CT system on which the PCD-CT was built.

Leng et $\mathrm{al}^{23}$ reported the preliminary results of PCD-CT UHR imaging using various phantom and cadaveric test objects. Among these studies, 1 cadaveric temporal bone was scanned, and it was found that the PCD-CT UHR acquisition with a servicemode sharp kernel (S80) achieved 29\% noise reduction compared with the EID-CT system. In this present work, with results from multiple cadaveric temporal bone specimens, we have demonstrated that with a clinical temporal bone reconstruction kernel (U70), the PCD-CT UHR mode could achieve $\sim 40 \%$ noise reduction compared with an EID-CT system when scanning at the same dose level and reconstructing at the same image thickness $(0.6 \mathrm{~mm})$. The more aggressive noise reduction with PCD in this study compared with the previous report ${ }^{23}$ is mainly due to the kernel difference (U70 is sharper than S80). Our results indicate the potential of a $64 \%$ reduction in dose using PCD-CT for clinical temporal bone imaging to achieve the same image noise as in EID-CT. This finding confirmed the previous conclusion that PCD-CT with its direct energy conversion could substantially increase the dose efficiency of UHR acquisitions compared with the EID-CT technique using a comb filter. ${ }^{29}$

Both PCD image sets (PCD-0.6 and PCD-0.25) were preferred compared with EID acquisitions because submillimeter structures were more evident on PCD images. One contribution was from the slightly improved in-plane resolution on the PCD-CT. At matched image thicknesses $(0.6 \mathrm{~mm}), \mathrm{PCD}-0.6$ images had the additional benefit of significantly lower image noise than the EID0.6 images. On the other hand, PCD-0.25 images had the benefit 


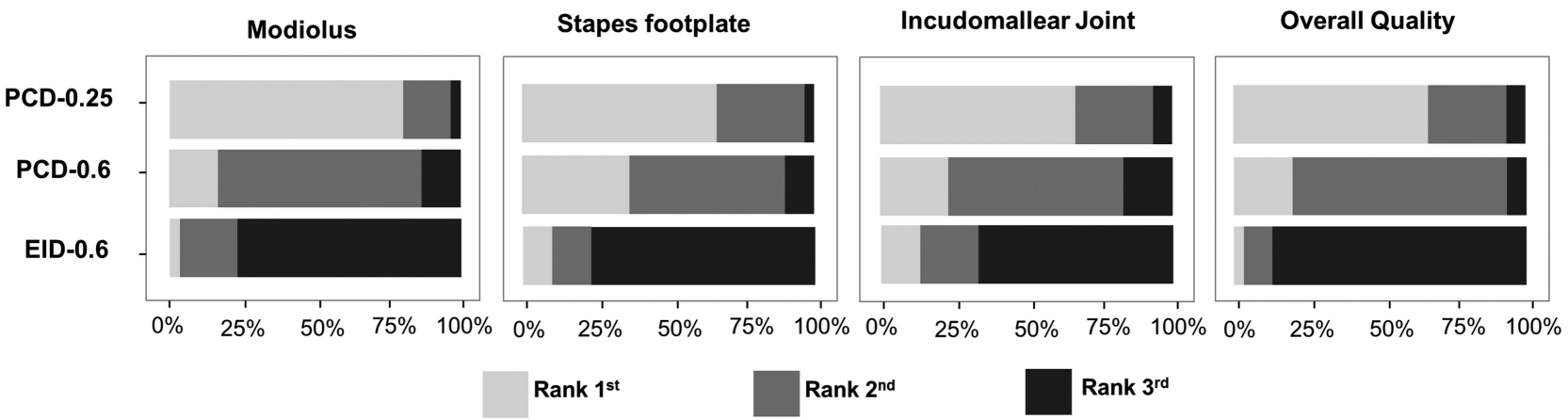

FIG 6. Rankings from 3 readers regarding overall image quality and delineation of 3 key anatomic structures. For all 3 structures and overall image quality, UHR PCD-CT images with 0.25-mm thickness have the highest rank (average, 1.2-1.4), while UHR EID-CT images with 0.6-mm thickness have the lowest rank (average, 2.5-2.8). White indicates the first rank; gray, the second rank; black, the third rank.

Table 2: Visual assessment and comparison of image quality on 3 image sets acquired from PCD- and EID-CT scanners

\begin{tabular}{lcccccccc}
\hline & \multicolumn{3}{c}{ Average Rank } & & \multicolumn{3}{c}{ Pair-Wise Comparison P Value } \\
\cline { 2 - 3 } & PCD-0.25 & PCD-0.6 & EID-0.6 & & PCD-0.25 vs PCD-0.6 & PCD-0.25 vs EID-0.6 & PCD-0.6 vs EID-0.6 \\
\hline Modiolus & 1.2 & 2.0 & 2.7 & & .002 & .001 & $<.001$ \\
Stapes footplate & 1.4 & 1.7 & 2.7 & & .12 & $<.001$ & $<.001$ \\
Incudomallear joint & 1.4 & 1.9 & 2.5 & & $<.001$ & $<.001$ & .02 \\
Overall quality & 1.4 & 1.9 & 2.8 & & $<.001$ & $<.001$ & $<.001$ \\
\hline
\end{tabular}

Table 3: Interobserver agreement among 3 neuroradiologists for image-quality assessment of the 10 temporal bone cases

\begin{tabular}{lccl}
\hline & K- $\boldsymbol{\alpha}$ & $\begin{array}{c}\text { Successful } \\
\text { Cases }\end{array}$ & $\begin{array}{c}\text { Interobserver } \\
\text { Agreement }\end{array}$ \\
\hline Modiolus & $0.54(0.37-0.69)$ & $7 / 10$ & Moderate \\
Stapes footplate & $0.44(0.27-0.59)$ & $7 / 10$ & Moderate \\
Incudomallear joint & $0.36(0.16-0.54)$ & $6 / 10$ & Fair \\
Overall quality & $0.50(0.33-0.67)$ & $7 / 10$ & Moderate \\
\hline
\end{tabular}

${ }^{a}$ Results were represented by K- $\alpha$ (95\% confidence level). The number of cases with ranking agreement from majority of raters $(\geq 2)$ was recognized as successful agreement cases.

of thinner images and less axial partial volume averaging compared with the EID-0.6 mm images, with no increase in image noise. Readers showed a strong preference for thinner PCD images despite their increased noise level.

Although this study focused on only temporal bone imaging, the demonstrated benefits may be applicable to other areas. For example, the use of thin CT images has been proved to increase the detectability of structural abnormalities in the temporal bone, ${ }^{30}$ coronary artery, ${ }^{31}$ and pulmonary nodules. ${ }^{32,33}$ Recently, a prototype whole-body system using an EID detector at a smaller (0.25-mm) detector cell was introduced for lung imaging. ${ }^{34}$ However, the loss of geometric efficiency from an increased density of septa substantially increased image noise. This is not an issue for PCD because no septa are required with its direct-conversion technique. Smaller image thicknesses reduce partial volume averaging and result in enhanced visualization of submillimeter structures.

In this study, the pyramid-shaped modiolus, the central bony pillar of the cochlea, was better depicted with the PCD system (Fig 2). The modiolus accommodates the fine terminal branches of the cochlear nerve, and hyperattenuation of the modiolus on CT can be observed in cases of cochlear nerve aplasia. ${ }^{2}$ Determination of cochlear nerve aplasia can prove critical in the evaluation of patients with profound sensorineural hearing loss because it would preclude the possibility of successful cochlear implantation. ${ }^{35}$
Similarly, a patulous modiolus is associated with intraoperative CSF leaks during cochlear implantation, and preoperative knowledge of this malformation would allow the surgeon to modify the approach accordingly. Likewise, in the evaluation of conductive hearing loss, confirming the integrity of the ossicular chain is critical to patient management. Our results suggest that PCD will be superior to EID in the detection of subtle ossicular abnormalities, including discontinuity, fibrous union, congenital fusion, and fixation (eg, otosclerosis, tympanosclerosis).

The PCD-CT scanner investigated in this study is a research system, and there is currently no commercial PCD-CT system available. More work is required to make the system more cost-effective and reliable, like the EID-CT systems, so that it can be used in routine clinical practice. This study represents the first step toward the adoption of the PCD-CT UHR mode for diagnostic neuroradiology practice. There were, however, several limitations to this study. First, this was an in vitro, cadaveric study. Cadaveric specimens were used because repeat scans could be easily performed in a well-controlled fashion. Because key anatomic structures were well-preserved in the cadaveric specimens, the results of our study represent what would be expected with patient imaging, though a future in vivo study is warranted. Second, the current study focused on image-quality assessment without evaluating diagnostic accuracy due to the lack of substantial pathology in the available cadaveric specimens. With these preliminary results showing the benefit of PCD-CT, our future studies will focus on diagnostic accuracy for in vivo patient examinations with specific pathology.

\section{CONCLUSIONS}

This study demonstrated, for the first time, the improvement in image quality and reader preference in temporal bone imaging using the UHR PCD-CT technology. With the superior inplane resolution and ultrathin $(0.25-\mathrm{mm})$ image thickness, the 
PCD-CT system demonstrated better delineation of anatomic microstructures of the temporal bones compared with UHR acquisitions performed on a commercial EID-CT system.

Disclosures: Cynthia H. McCollough—RELATED: Grant: Siemens*; Provision of Writing Assistance, Medicines, Equipment, or Administrative Support: Siemens *; UNRELATED: Grants/Grants Pending: Siemens, Bayer Healthcare*; Royalties: Bayer Healthcare.* Shuai Leng_RELATED: Grant: National Institutes of Health, Comments: Research reported in this article was supported by the National Institutes of Health under award numbers R01 EB016966 and C06 RR018898 and in collaboration with Siemens*. *Money paid to the institution.

\section{REFERENCES}

1. Zayas JO, Feliciano YZ, Hadley CR, et al. Temporal bone trauma and the role of multidetector CT in the emergency department. Radiographics 2011;31:1741-55 CrossRef Medline

2. Lane JI, Lindell EP, Witte RJ, et al. Middle and inner ear: improved depiction with multiplanar reconstruction of volumetric CT data. Radiographics 2006;26:115-24 CrossRef Medline

3. Majdani O, Thews K, Bartling S, et al. Temporal bone imaging: comparison of flat panel volume CT and multisection CT. AJNR Am J Neuroradiol 2009;30:1419-24 CrossRef Medline

4. Jäger L, Bonell H, Liebl M, et al. CT of the normal temporal bone: comparison of multi- and single-detector row CT. Radiology 2005; 235:133-41 CrossRef Medline

5. Goldfeld M, Glaser B, Nassir E, et al. CT of the ear in Pendred syndrome. Radiology 2005;235:537-40 CrossRef Medline

6. Flohr TG, Stierstorfer K, Süss C, et al. Novel ultrahigh resolution data acquisition and image reconstruction for multi-detector row CT. Med Phys 2007;34:1712-23 Medline

7. McCollough CH, Leng S, Sunnegardh J, et al. Spatial resolution improvement and dose reduction potential for inner ear CT imaging using a z-axis deconvolution technique. Med Phys 2013;40:061904 CrossRef Medline

8. Meyer M, Haubenreisser H, Raupach $\mathrm{R}$, et al. Initial results of a new generation dual source CT system using only an in-plane comb filter for ultra-high-resolution temporal bone imaging. Eur Radiol 2015;25:178-85 CrossRef Medline

9. Leng S, Diehn FE, Lane JI, et al. Temporal bone CT: improved image quality and potential for decreased radiation dose using an ultrahigh-resolution scan mode with an iterative reconstruction algorithm. AJNR Am J Neuroradiol 2015;36:1599-603 CrossRef Medline

10. Gupta R, Grasruck M, Suess C, et al. Ultra-high resolution flat-panel volume CT: fundamental principles, design architecture, and system characterization. Eur Radiol 2006;16:1191-205 CrossRef Medline

11. Kalender WA, Kyriakou Y. Flat-detector computed tomography (FD-CT). Eur Radiol 2007;17:2767-79 CrossRef Medline

12. Kappler S, Hannemann $\mathrm{T}$, Kraft E, et al. First results from a hybrid prototype CT scanner for exploring benefits of quantum-counting in clinical CT. Proc SPIE 2012;8313:30 CrossRef

13. Kappler S, Henning A, Kreisler B, et al. Photon counting CT at elevated X-ray tube currents: contrast stability, image noise and multi-energy performance. Proc SPIE 2014;9033:90331C CrossRef

14. Yu Z, Leng S, Jorgensen SM, et al. Evaluation of conventional imaging performance in a research whole-body CT system with a photon-counting detector array. Phys Med Biol 2016;61:1572-95 CrossRef Medline

15. Schlomka JP, Roessl E, Dorscheid R, et al. Experimental feasibility of multi-energy photon-counting K-edge imaging in pre-clinical computed tomography. Phys Med Biol 2008;53:4031-47 CrossRef Medline

16. Pourmorteza A, Symons R, Sandfort V, et al. Abdominal imaging with contrast-enhanced photon-counting CT: first human experience. Radiology 2016;279:239-45 CrossRef Medline

17. Shikhaliev PM. Energy-resolved computed tomography: first experimental results. Phys Med Biol 2008;53:5595-613 CrossRef Medline

18. Iwanczyk JS, Nygard E, Meirav O, et al. Photon counting energy dispersive detector arrays for X-ray imaging. IEEE Trans Nucl Sci 2009;56:535-42 CrossRef Medline

19. Taguchi K, Iwanczyk JS. Vision 20/20: single photon counting $\mathbf{x}$-ray detectors in medical imaging. Med Phys 2013;40:100901 CrossRef Medline

20. Symons R, Pourmorteza A, Sandfort V, et al. Feasibility of dosereduced chest $\mathrm{CT}$ with photon-counting detectors: initial results in humans. Radiology 2017;285:980-89 CrossRef Medline

21. Pourmorteza A, Symons R, Reich D, et al. Photon-counting CT of the brain: in vivo human results and image-quality assessment. AJNR Am J Neuroradiol 2017;38:2257-63 CrossRef Medline

22. Symons R, Reich DS, Bagheri M, et al. Photon-counting computed tomography for vascular imaging of the head and neck: first in vivo human results. Invest Radiol 2018;53:135-42 CrossRef Medline

23. Leng S, Yu Z, Halaweish A, et al. Dose-efficient ultrahigh-resolution scan mode using a photon counting detector computed tomography system. J Med Imaging (Bellingham) 2016;3:043504 CrossRef Medline

24. Zhou W, Montoya J, Gutjahr R, et al. Lung nodule volume quantification and shape differentiation with an ultra-high-resolution technique on a photon counting detector CT system. In: Proceedings of SPIE Medical Imaging 2017: Physics of Medical Imaging. Orlando, Florida; June 5, 2017

25. Schardt P, Deuringer J, Freudenberger J, et al. New x-ray tube performance in computed tomography by introducing the rotating envelope tube technology. Med Phys 2004;31:2699-706 CrossRef Medline

26. Schuknecht H. Pathology of the Ear. Boston: Harvard University Press; 1975

27. Norweck JT, Seibert JA, Andriole KP, et al. ACR-AAPM-SIIM technical standard for electronic practice of medical imaging. J Digit Imaging 2013;26:38-52 CrossRef Medline

28. Landis JR, Koch GG. Measurement of observer agreement for categorical data. Biometrics 1977;33:159-74 CrossRef Medline

29. Leng S, Gutjahr R, Ferrero AF, et al. Ultra-high spatial resolution, multi-energy CT using photon counting detector technology. Proc SPIE Int Soc Opt 2017;10132:101320Y CrossRef

30. Caldemeyer KS, Sandrasegaran K, Shinaver CN, et al. Temporal bone: comparison of isotropic helical CT and conventional direct axial and coronal CT. AJR Am J Roentgenol 1999;172:1675-82 CrossRef Medline

31. Nieman K, Cademartiri F, Lemos PA, et al. Reliable noninvasive coronary angiography with fast submillimeter multislice spiral computed tomography. Circulation 2002;106:2051-54 CrossRef Medline

32. Petrou M, Quint LE, Nan B, et al. Pulmonary nodule volumetric measurement variability as a function of CT slice thickness and nodule morphology. AJR Am J Roentgenol 2007;188:306-12 CrossRef Medline

33. Kawel N, Seifert B, Luetolf M, et al. Effect of slab thickness on the CT detection of pulmonary nodules: use of sliding thin-slab maximum intensity projection and volume rendering. $A J R A m$ J Roentgenol 2009;192:1324-29 CrossRef Medline

34. Kakinuma R, Moriyama N, Muramatsu Y, et al. Ultra-high-resolution computed tomography of the lung: image quality of a prototype scanner. PLoS One 2015;10:e0137165 CrossRef Medline

35. Glastonbury CM, Davidson HC, Harnsberger HR, et al. Imaging findings of cochlear nerve deficiency. AJNR Am J Neuroradiol 2002; 23:635-43 Medline 